\title{
Towards Intra-operative Prostate Brachytherapy Dosimetry Based on Partial Seed Localization in Ultrasound and Registration to C-arm Fluoroscopy
}

\author{
Mehdi Moradi ${ }^{1, \star}$, S. Sara Mahdavi ${ }^{1}$, Sanchit Deshmukh ${ }^{2}$, Julio Lobo ${ }^{1}$, \\ Ehsan Dehghan ${ }^{3}$, Gabor Fichtinger ${ }^{3}$, \\ William J. Morris ${ }^{4}$, and Septimiu E. Salcudean ${ }^{1, \star}$ \\ 1 Department of Electrical and Computer Engineering, \\ University of British Columbia, Vancouver, Canada \\ \{moradi, saram, juliol, tims\}@ece.ubc.ca \\ 2 Indian Institute of Technology, Bombay, India \\ 3 School of Computing, Queen's University, Kingston, ON, Canada \\ 4 British Columbia Cancer Agency
}

\begin{abstract}
Intraoperative dosimetry during prostate brachytherapy is a long standing clinical problem. We propose a novel framework to address this problem by reliable detection of a subset of seeds from 3D transrectal ultrasound and registration to fluoroscopy. Seed detection in ultrasound is achieved through template matching in the RF ultrasound domain followed by thresholding and spatial filtering based on the fixed distance between stranded seeds. This subset of seeds is registered to the complete reconstruction of the implant in C-arm fluoroscopy. We report results, validated with a leave-one-needle-out approach, both in a phantom (average post-registration seed distance of $2.5 \mathrm{~mm}$ ) and in three clinical patient datasets (average error: $3.9 \mathrm{~mm}$ over 113 seeds).
\end{abstract}

\section{Introduction}

Low dose rate brachytherapy is a minimally invasive therapeutic procedure for prostate cancer that has rapidly gained acceptance due to highly successful clinical results. In this procedure, a number of small radioactive sources or seeds $\left({ }^{125} \mathrm{I}\right.$ or ${ }^{103} \mathrm{Pd}$ ) are permanently implanted into the prostate using brachytherapy needles. The aim is to deliver a sufficient radiation dose to kill cancerous tissue while limiting the dose in radio-sensitive regions such as the bladder, urethra and rectum. Transrectal ultrasound (TRUS) is used to intraoperatively guide the transperineal insertion of needles. As a result of prostate edema, motion of the gland due to needle forces, and possible intra-operative changes to the plan due to various factors such as interference with the pubic arch, the locations of the implanted seeds do not necessarily match with the initial treatment plan. Hence, for quality assurance, intra-operative dosimetry is highly beneficial.

\footnotetext{
^ Corresponding authors.
} 
Researchers have approached ultrasound-based seed detection [1]. However, accurate seed localization based on ultrasound has proven to be a very difficult task due to clutter from other highly reflecting objects such as calcifications resulting in false positive appearances, seed specularity and shadowing, and limited field of view. Even when hand-segmented, up to $25 \%$ of the seeds remain hidden in ultrasound images [2]. Therefore, C-arm fluoroscopy is commonly used for visual assessment of the implanted seeds. However, the prostate gland is not discernible in fluoroscopy images. Fusion of the fluoroscopy images and ultrasound is therefore considered as a possible solution [3 6]. If complete seed localization and implant reconstruction from fluoroscopy images is available, the registration of the result to ultrasound will enable dosimetry. In recent years, the fluoroscopy reconstruction problem has been extensively addressed. Given 3-5 fluoroscopy images, and the relative pose of the $\mathrm{C}$-arm in each acquisition, the back-projection technique can be used to reconstruct the $3 \mathrm{D}$ implant 7]. For registration, attaching fiducial markers to the ultrasound probe [8], using the ultrasound probe itself as a fiducial [4], or using the seeds as fiducials [9] have been mentioned. Due to patient and equipment motion between the acquisition of ultrasound and fluoroscopy, registration based on static markers is not reliable. Furthermore, the use of fiducial markers is an unwelcome addition to the ordinary setup in the operating room due to time and space limitations.

In this work we propose using a subset of seeds extracted from ultrasound images to perform point-based registration between the seed clouds from fluoroscopy and 3D ultrasound. We present several technical innovations. Instead of conventional B-mode ultrasound, we use RF signals processed to enhance seed contrast. Template matching with a variety of in vivo and ex vivo seed templates is reported. To enable dosimetry, we have devised a two stage strategy consisting of first $2 \mathrm{D}$ registration of needle projections from the ultrasound and fluoroscopy, followed by the 3D registration of only the seeds in the matched needles. We provide the results of this approach on both clinical and phantom data. This novel methodology targets a complicated and long standing problem, with no addition to the routine therapeutic procedure. We show that our method has good promise to address this clinical challenge.

\section{Methods}

The outline of our methodology is presented in Figure 1, For reconstruction of the implant in fluoroscopy, we implemented and used the method described in [7]. The steps for acquiring the fluoroscopy reconstruction included C-arm pose estimation from rotation angle and compensation for sagging, followed by backprojection of the seeds, and finally seed to needle assignment using a minimum cost flow (MCF) algorithm. The outcome was validated in terms of number of seeds, needles, and seed to needle assignments based on the brachytherapy plans, both in patient and phantom datasets. In this article we focus on ultrasoundbased partial seed detection, needle matching and registration from ultrasound to fluoroscopy. 


\subsection{Seed Detection}

3D Ultrasound Setup and Data: We developed a 3D ultrasound system based on a brachytherapy stepper (EXII, CIVCO Medical Solutions) modified by motorizing the cradle rotation. The sagittal array of a dual plane linear/microconvex broadband 5-9 MHz endorectal transducer (Ultrasonix) was used. $\mathrm{RF}$ data was recorded at a frame rate of $42 \mathrm{fps}$, during the probe rotation from $-45^{\circ}$ to $50^{\circ}\left(0^{\circ}\right.$ corresponded to the probe aligned with the central sagittal cross section of the prostate gland). $2 \mathrm{D}$ frame size was $5 \times 5.5 \mathrm{~cm}$. We present the results of our work on data collected immediately after seed implantation in the OR, from three brachytherapy patients in Vancouver Cancer Center. We also present data from a CIRS Model 053 tissue-equivalent ultrasound prostate phantom (CIRS, Inc., Norfolk, VA). For this phantom, a plan consisting of 135 seeds and 26 needles was created which was carried out by a radiation oncologist.

RF Signal Processing: In order to improve the seed to background contrast, we averaged the signal power over windowed blocks of the RF signals. In other words, we replaced a segment of length $n$ at depth $d$ of an RF line with the reflected power $P_{d}$ computed as:

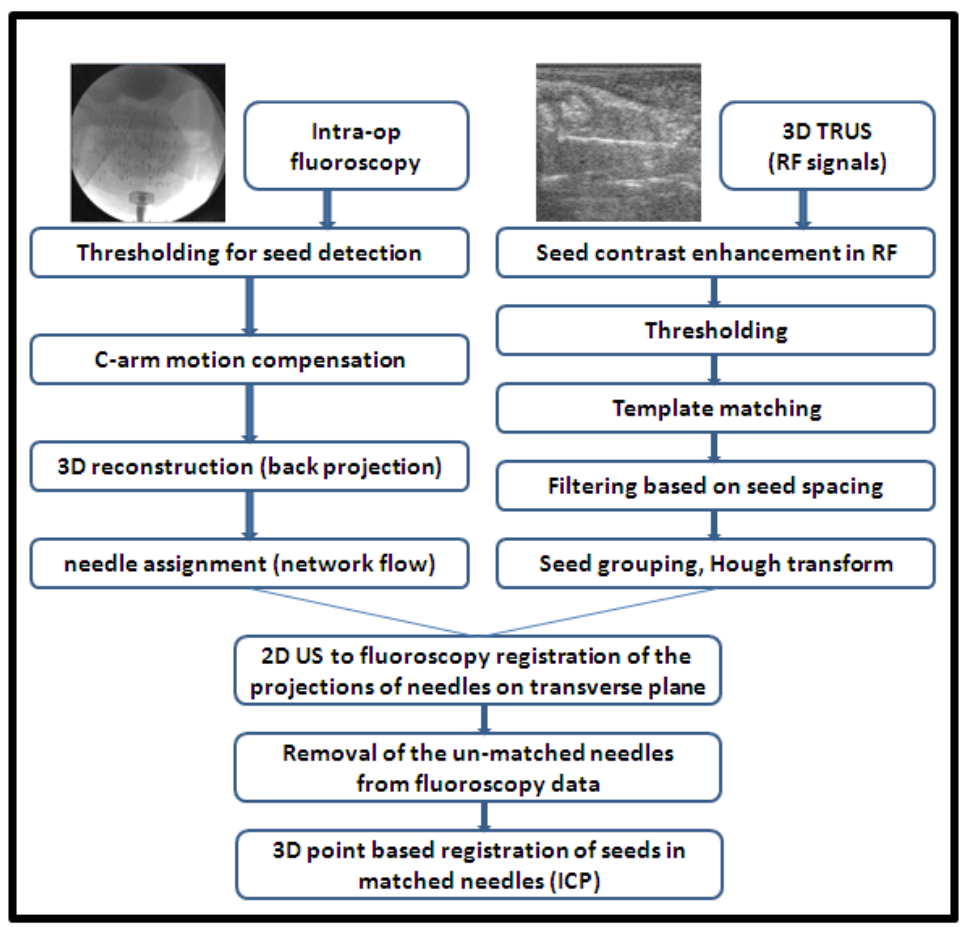

Fig. 1. Workflow of the seed detection method 


$$
P_{d}=\frac{\sum_{k=1}^{n} w(k) x(k)^{2}}{n}
$$

where $x(k)(k=1, \ldots, n)$ are the samples in the RF segment, and $w(k)$ are Hamming window weights. This step was applied with $n=10$ and a $50 \%$ overlap between consecutive blocks. We have previously reported that this process doubles the contrast to noise ratio (CNR) between the seed regions and the background [10]. Furthermore, the resulting five fold reducion in the size of the RF signals improved the speed of the template matching step.

Seed Templates: Simple thresholding of the contrast enhanced RF data results in a large number of seed candidates and seed-like clutter. In order to reduce false positive detections, we computed the normalized cross correlation of the seed regions with seed templates. We experimented with three groups of templates and will provide a comparison.

Ex vivo templates: We created a 3D template acquired by imaging a seed in water, placed parallel to the probe at the center of the ultrasound probe. The $3 \mathrm{D}$ template was formed by rotating the probe and acquiring 21 sagittal images.

In vivo templates: In the clinical situation, the existence of background tissue, blood and edema significantly alters the appearance of seeds on ultrasound images compared to the described ex vivo templates, resulting in low normalized cross correlation values. Therefore, we created a second set of templates extracted from in vivo data. These were extracted from different cases to ensure that the template extracted from a specific patient dataset, was not used for seed detection in that dataset. They were acquired by manually clicking the center of a visually distinct seed in in vivo $3 \mathrm{D}$ data.

In vivo and ex vivo two-seed templates: We also created templates, both in vivo and ex vivo, in which the template area contained two seeds. The twoseed templates were examined as a potential solution to reduce the number of false positives, given the fact that in stranded brachytherapy performed in our institution, the distance between seeds is fixed at $1 \mathrm{~cm}$ with very few exceptions. Therefore, the existence of two strong seed candidates, $1 \mathrm{~cm}$ apart, is an indication of a true detection.

NCC-Based 3D Template Matching: The normalized cross correlation of the $3 \mathrm{D}$ template $g$ and a cropped area of the image $f$ equal in size with $g$ and centered at location $(i, j, k)$ can be computed as:

$$
f \star g(i, j, k)=\frac{\left(\sum_{x, y, z} f_{i, j, k}(x, y, z)-\overline{f_{i, j, k}}\right) \cdot(g(x, y, z)-\bar{g})}{\sqrt{\left[\sum_{x, y, z}\left(f_{i, j, k}(x, y, z)-\overline{f_{i, j, k}}\right)^{2} \sum_{x, y, z}(g(x, y, x)-\bar{g})^{2}\right]}}
$$

where $x, y$ and $z$ represent the directions in the image coordinate system. Computation of $f \star g$ results in a new image of the same size as $f$, with values in range of $[0,1]$ with largest values representing the centers of areas most similar to the template. The frequency domain implementation of NCC was completed in under six minutes on a regular PC with MATLAB for template size of $30 \times 60 \times 21$, and 
image size of $128 \times 258 \times 391$. Note that NCC is not invariant to scale. However, in our case, the images and the templates were acquired with similar imaging parameters, therefore the scales matched.

Thresholding, Spatial Filtering, and Grouping in the NCC Image: The NCC image was thresholded. Starting from the point with the highest NCC, a neighborhood of the size of the seed was cleared around each non-zero voxel. This was necessary because each seed consists of several bright voxels, while we need a single voxel to represent the seed. The remaining voxels were grouped into needles using the Hough transform [11] followed by eliminating single seeds that could not be grouped into lines. Using the knowledge of the fixed $1 \mathrm{~cm}$ distance between the seeds in our data, we applied an additional trimming step. On each needle, starting from the seed with the highest NCC value, any other seed candidate that was within $0.8 \mathrm{~cm}$ was removed.

\subsection{Matching and Registration}

After applying a transformation that matched the centers of mass of the two datasets, we applied a 2D needle matching process. This provided an initial alignment and reduced the risk of local minima due to the unbalanced number of seeds in ultrasound and fluoroscopy. Matching was performed by applying a 2D rigid registration between the needle projections on the transverse plane passing through the prostate mid-gland in the fluoroscopy implant. Assuming that $X$ is the set of $n$ projection points from ultrasound, and $Y$ is the set of $m$ projection points from fluoroscopy, the rotation and translation parameters of the transformation $T$ were found to minimize $\sum_{i=1: n} d_{c}\left[T\left(X_{i}\right), Y\right]$ where $d_{c}\left[T\left(X_{i}\right), Y\right]$ is the Euclidean distance of the ultrasound projection point $X_{i}$ from its closest match in the point set $Y$. After the matching step, the fluoroscopy needles without a match in ultrasound were removed and the standard 3D point-based Iterative Closest Point (ICP) registration algorithm [12] was applied.

\section{Results and Conclusions}

We quantified the outcome of our ICP seed registration method based on 1) the post-registration distances between ultrasound seeds and their closest fluoroscopy counterparts, 2) the stability of needle matches and the recorded registration errors subject to the removal of any of the detected needles.

Registration Errors: Table 1 presents the results of ultrasound seed detection and registration for the CIRS phantom. The best outcome was achieved when the two-seed ex vivo template set was used. The post-registration seed distances from ultrasound to the closest matching fluoroscopy seed was $2.48 \mathrm{~mm}$. Note that the best registration result was obtained when the lowest number of seeds ( 73 out of 135,17 needles out of 26 ) were detected. The result of the $2 \mathrm{D}$ matching and $3 \mathrm{D}$ registration of the seed clouds for the phantom data, using the two-seed ex vivo template, is depicted in Figure 2 . 

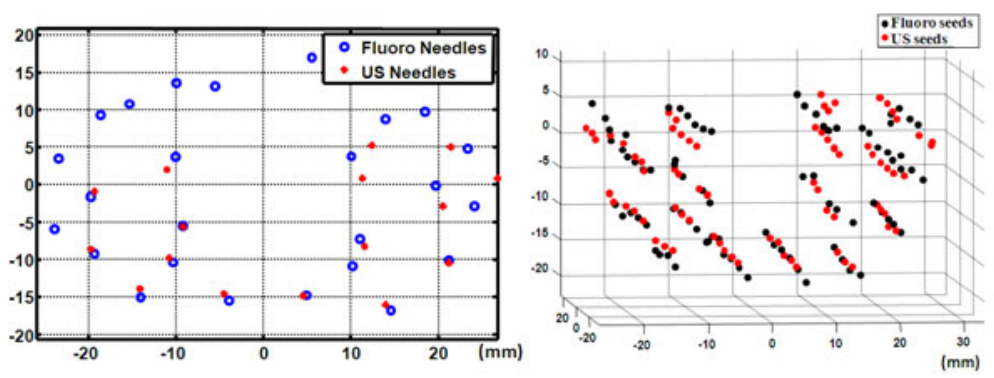

Fig. 2. Phantom results: 2D matching of needle projections in transverse plane (left), $3 \mathrm{D}$ seed cloud registration using the matched needles from fluoroscopy and ultrasound

Table 1. Phantom result: \# of detected seeds, registration errors with all detected needles, and average registration error for the leave-one-needle-out validation test

\begin{tabular}{|c|c|c|c|c|c|}
\hline Phantom & $\begin{array}{c}\text { dist. er. }(\mathrm{mm}) \\
\text { all needles }\end{array}$ & $\begin{array}{c}\text { dist. er. } \\
(\mathrm{mm}) \\
\text { L.O.O }\end{array}$ & $\begin{array}{c}\# \text { fl } \\
\text { seeds } \\
\text { Total }\end{array}$ & $\begin{array}{c}\text { \# fl seeds, } \\
\text { in matching } \\
\text { needles }\end{array}$ & $\begin{array}{c}\text { \# of detected } \\
\text { seeds in } \\
\text { ultrasound }\end{array}$ \\
\hline \hline 1-seed, ex vivo templ. & $2.69 \pm 2.03$ & $2.71 \pm 1.98$ & 135 & 95 & 86 \\
1-seed, in vivo templ. & $4.33 \pm 2.21$ & $4.40 \pm 2.24$ & 135 & 101 & 95 \\
2-seed, ex vivo templ. & $2.48 \pm 1.52$ & $2.48 \pm 1.41$ & 135 & 82 & 73 \\
\hline
\end{tabular}

Table 2 presents the results for the three patient datasets. The one-seed and the two-seed templates did not result in significantly different error values. We obtained errors of $3.36 \mathrm{~mm}, 3.73 \mathrm{~mm}$, and $4.76 \mathrm{~mm}$ for the three cases on in vivo templates. With ex vivo templates, we witnessed a decrease in the number of detected seeds for all cases and an error increase in two patients with a slight improvement in case 3 in terms of registration error (to 4.38, 4.08 and $4.22 \mathrm{~mm}$ ). For case 2, Figure 3 illustrates the results of 2D matching and 3D registration. In both Figures 2 and 3 one can see that the unmatched needles tend to be from the anterior side (top of the images), while the posterior seeds that are closest to the probe are accurately detected. This is likely due to signal attenuation and the depth dependent reduction in the resolution of our 3D ultrasound system.

Leave-one-needle-out Validation of the Registration Process: In order to study the stability of our matching and registration procedure, we also ran a leave-one-needle-out experiment. For each patient and the phantom case, assuming that $n$ ultrasound needles were identified, we repeated the matching and registration procedure $n$ times, each time with $n-1$ needles. This amounted to the removal of three to seven $(10 \%$ to $20 \%$ ) of the seeds in each round for patient cases. The idea is that if the registration is valid, and not just a local minimum, the removal of any specific needle should not drastically change the outcome.

The average of the errors in the leave one out experiments (column 3 in Tables 1 and 21) were close to the errors when all detected seeds were used in the registration step (column 2). We also examined the stability of the needle 

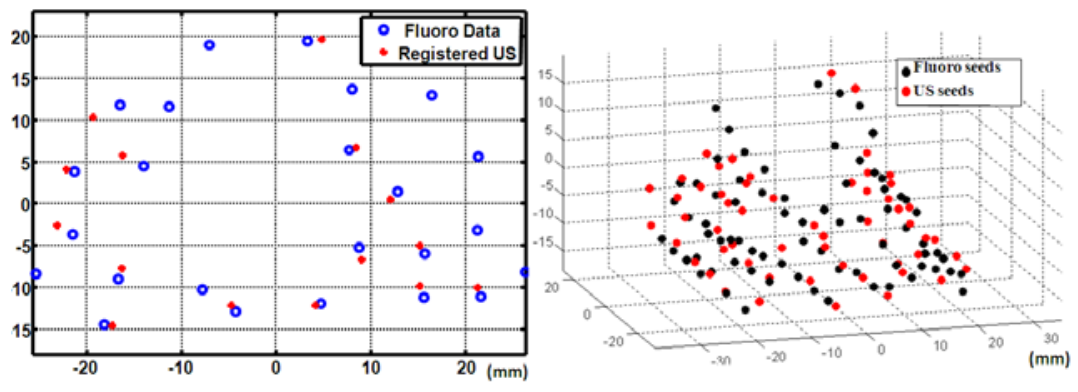

Fig. 3. A patient case: $2 \mathrm{D}$ matching (left), registered seeds in matched needles (right)

Table 2. Number of the detected seeds, registration errors and leave-one-needle-out errors for the 3 patient datasets

\begin{tabular}{|c|c|c|c|c|c|}
\hline Case & $\begin{array}{c}\text { dist. er. }(\mathrm{mm}) \\
\text { all needles }\end{array}$ & $\begin{array}{c}\text { dist. } \text { er. } \\
(\mathrm{mm}) \\
\text { L.O.O }\end{array}$ & $\begin{array}{c}\# \mathrm{fl} \\
\text { seeds } \\
\text { Total }\end{array}$ & $\begin{array}{c}\text { \# fl seeds, } \\
\text { in matching } \\
\text { needles }\end{array}$ & $\begin{array}{c}\text { \# of detected } \\
\text { seeds in } \\
\text { ultrasound }\end{array}$ \\
\hline P1 1-seed in vivo templ. & $3.72 \pm 1.86$ & $3.85 \pm 1.96$ & 102 & 51 & 35 \\
P1 2-seed in vivo templ. & $3.36 \pm 1.78$ & $3.44 \pm 1.95$ & 102 & 57 & 37 \\
P1 ex vivo templ. & $4.07 \pm 2.10$ & $4.38 \pm 1.87$ & 102 & 30 & 16 \\
\hline P2 1-seed in vivo templ. & $3.98 \pm 2.50$ & $3.77 \pm 2.27$ & 115 & 74 & 56 \\
P2 2-seed in vivo templ. & $3.73 \pm 1.86$ & $3.79 \pm 1.94$ & 115 & 71 & 49 \\
P2 ex vivo templ. & $3.74 \pm 2.8$ & $4.08 \pm 2.58$ & 115 & 55 & 37 \\
\hline P3 1-seed in vivo templ. & $4.76 \pm 1.87$ & $4.90 \pm 2.12$ & 100 & 35 & 27 \\
P3 2-seed in vivo templ. & $4.88 \pm 1.95$ & $4.79 \pm 2.25$ & 100 & 33 & 23 \\
P3 ex vivo templ. & $4.52 \pm 1.65$ & $4.22 \pm 1.49$ & 100 & 26 & 16 \\
\hline
\end{tabular}

matching step subject to removal of needles. In the case of the phantom data, and case 1 among the patient datasets, it was noted that regardless of which needle was removed in the leave one out experiment, the matched fluoroscopy needle for the rest of ultrasound needles remained the same (number of detected needles: $n=17$ ). In patient cases 2 and 3 , on average, the match for one needle changed due to the removal of a needle $(n=14$ and $n=12)$.

\section{Conclusions}

We showed that it is feasible to use contrast enhanced RF ultrasound data, template matching, and spatial filtering to detect a reliable subset of brachytherapy seeds from ultrasound to enable registration to fluoroscopy. Real-time implementation requires the matching process to be computationally improved though parallelization to enhance the current computation time of around six minutes. More robustness analysis and additional registration approaches will be implemented in future work. The 3D ultrasound system used in this work acquired sagittal images while rotating radially. This results in a significant decrease in 
spatial resolution at increasing distances from the probe. The use of a comprehensive depth dependent set of templates can improve our results. It is also possible to perform the ultrasound seed detection after only a part of the implant, for example the top row of needles, is completed. This will reduce the shadowing effects. Clinical data is being acquired to test these possibilities.

Acknowledgments. Dr T. Pickles and Mr N. Chng (data acquisition); Dr X. Wen (RP imaging codes). M. Moradi held an NSERC PDF and the US DoD PCRP Award W81XWH-10-1-0201, S. Mahdavi was supported by the Prostate Cancer Foundation of BC, G. Fichtinger was supported as Cancer Care Ontario Research Chair, S. E. Salcudean was supported by NSERC and CIHR.

\section{References}

1. Mamou, J., Ramachandran, S., Feleppa, E.J.: Angle-dependent ultrasonic detection and imaging of brachytherapy seeds using singular spectrum analysis. Journal of the Acoustical Society of America 123(4), 2148-2159 (2008)

2. Jain, A.K., Zhou, Y., Mustufa, T., Burdette, E.C., Chirikjian, G.S., Fichtinger, G.: Matching and reconstruction of brachytherapy seeds using the hungarian algorithm (MARSHAL). In: SPIE Medical Imaging. SPIE, vol. 5744, pp. 810-821 (2005)

3. Fallavollita, P., Karim-Aghaloo, Z., Burdette, C.E., Song, D.Y., Abolmaesumi, P., Fichtinger, G.: Registration between ultrasound and fluoroscopy or CT in prostate brachytherapy. Med. Phys. 37(6), 2749-2760 (2010)

4. French, D., Morris, J., Keyes, M., Salcudean, S.E.: Real-time dosimetry for prostate brachytherapy using TRUS and fluoroscopy. In: Barillot, C., Haynor, D.R., Hellier, P. (eds.) MICCAI 2004. LNCS, vol. 3217, pp. 983-991. Springer, Heidelberg (2004)

5. Su, Y., Davis, B.J., Furutani, K.M., Herman, M.G., Robb, R.A.: Seed localization and trus-fluoroscopy fusion for intraoperative prostate brachytherapy dosimetry. Comput. Aided Surg. 12(1), 25-34 (2007)

6. Todor, D.A., Zaider, M., Cohen, G.N., Worman, M.F., Zelefsky, M.J.: Intraoperative dynamic dosimetry for prostate implants. Physics in Medicine and Biology 48, 1153-1171 (2003)

7. Dehghan, E., Lee, J., Moradi, M., Wen, X., Fichtinger, G., Salcudean, S.E.: Prostate brachytherapy seed reconstruction using C-arm rotation measurement and motion compensation. In: Jiang, T., Navab, N., Pluim, J.P.W., Viergever, M.A. (eds.) MICCAI 2010. LNCS, vol. 6361, pp. 283-290. Springer, Heidelberg (2010)

8. Zhang, M., Zaider, M., Worman, M., Cohen, G.: On the question of 3D seed reconstruction in prostate brachytherapy: the determination of x-ray source and film locations. Phys. Med. Biol. 49(19), N335-N345 (2004)

9. Tutar, I.B., Gong, L., Narayanan, S., Pathak, S.D., Cho, P.S., Wallner, K., Kim, Y.: Seed-based transrectal ultrasound-fluoroscopy registration method for intraoperative dosimetry analysis of prostate brachytherapy. Med. Phys. 35(3), 840-848 (2008)

10. Wen, X., Salcudean, S.E., Lawrence, P.D.: Detection of brachytherapy seeds using 3-D transrectal ultrasound. IEEE Trans. Biomed. Eng. 57(10), 2467-2477 (2010)

11. Duda, R.O., Hart, P.E.: Use of the Hough transformation to detect lines and curves in pictures. Communications of the ACM 15, 11-15 (1972)

12. Besl, P.J., McKay, N.D.: A method for registration of 3-D shapes. IEEE Transactions on Pattern Analysis and Machine Intelligence 14(2), 239-256 (1992) 